\title{
Formulación y Control de la Estrategia en un Grupo de Empresas que Conforman una Red
}

\author{
Merlin Patricia Grueso H. ', Jorge Hernán Gómez ${ }^{2}$, Leonardo Garay ${ }^{3}$
}

\begin{abstract}
The management's networks are part of the actual business surrounding and characterize themselves because they promote the establishment of a strong interdependency between the companies, their clients, suppliers, government institutions and supporting entities. The investigation proposal was to evaluate the degree in which the enterprises include these actors in the formulation and control of the management's strategy The results shows that the companies involved are taking especially into consideration the interaction with their employees at the moment to formulate and realize controls of managements' strategy. The involvement of the other enterprises which adjusts the network in this procedure is questionable; at the time the importance that the enterprises ascribe to universities and entities who promote investigation, is limited.
\end{abstract}

Keywords: strategy formulation; strategy control; enterprise's networks.

\section{Resumen}

Las redes empresariales son parte del actual entorno de negocios y se caracterizan porque llevan a establecer fuertes interdependencias entre las empresas, sus clientes, proveedores, instituciones del gobierno y entidades de apoyo. El estudio tenía como propósito validar el grado en que las empresas consideran estos agentes en los procesos de formulación y control de la estrategia empresarial. Los resultados obtenidos indican que las empresas tienen en cuenta especialmente la interacción con sus empleados al momento de formular y realizar controles a las estrategias empresariales; el papel de las otras empresas que conforman la red en este proceso es discutible; así mismo, es limitada la importancia que las empresas le atribuyen a las universidades y entidades de fomento a la investigación.

Palabras clave: formulación de estrategia; control de estrategia; redes empresariales,

\footnotetext{
' Universidad del Rosario, Facultad de Administración. Universidad del Rosario. Calle 14 \# 4-69. Bogotá Colombia. Tel: 0057(I)2970200 Ext 7837. Email: merlin.grueso@urosario.edu.co

${ }^{2}$ Universidad del Rosario, Facultad de Administración, Director CIDEM, Carrera $7^{\text {a }}$ No 13 4I of. 80I, Teléfono 282805I, BogotáColombia. Email: jhgomez@urosario.edu.co

${ }^{3}$ Universidad del Rosario, CIDEM, Asistente de Proyecto de Redes Empresariales, Carrera $7^{\text {a }}$ No 13 4I of. 80I, Teléfono 282805I, Bogotá-Colombia. Email: leonardo.garay@urosario.edu.co
} 


\section{Introducción}

Las empresas no trabajan en solitario (Barabási, 2003). El actual entorno de negocios se caracteriza por un entramado de relaciones formales e informales entre diferentes agentes lo que da lugar a lo que se ha denominado "red de empresa" (Lipnack y Stamps, 1994).

Las redes de empresa se caracterizan por la interdependencia de las organizaciones con sus clientes y proveedores así como también por los vínculos que establece y mantiene con instituciones financieras, entidades del gobierno, universidades, competidores, entidades públicas y privadas (Eng, 2005).

En torno a las redes empresariales se han elaborado varias definiciones. Una de las más clásicas aproximaciones al concepto es desarrollada por Cook y Emerson (1978) quienes definen una red de empresas como aquella que se conforma a partir de las relaciones que se establecen en un conjunto de dos o más negocios.

Una definición más contemporánea, desarrollada en torno a los elementos fundamentales del concepto, es elaborada por López (2003), quien define las redes empresariales como "una alianza estratégica permanente entre un grupo limitado y claramente definido de empresas independientes, que colaboran para alcanzar objetivos comunes de mediano y largo plazo, orientados hacia el desarrollo de la competitividad de los distintos participantes" (p.22).

Pese a las distintas aproximaciones que existen en torno al concepto, todas coinciden en que las redes empresariales se constituyen en una estrategia colectiva que permite la resolución de problemas conjuntos. Con base en los desarrollos alcanzados sobre el tema se ha identificado también que una de sus principales características es que si bien las empresas que constituyen una red de empresa cooperan entre sí, mantienen su autonomía como ente organizacional.

La conformación de redes de empresa es un fenómeno que cada vez cobra mayor fuerza tanto en países desarrollados como en vías de desarrollo, debido a su probada relación con indicadores de desempeño organizacional como es el aumento en ventas colectivas (Ceglie y Dini, 1999), mejora de la productividad (Díaz, 2005; Frías y Rodríguez, 2005), disminución de costos de adquisición de materiales e insumos (López, 2003) e introducción de innovaciones en las organizaciones (Dini, et al., 2007).

Otra de las ventajas asociadas a la conformación de redes es que a través de ellas las organizaciones pueden afrontar condiciones altamente complejas (Lipnack y Stamps, 1994) que en solitario, no estarían en capacidad de encarar de manera óptima.

El establecimiento de contactos colaborativos de carácter formal e informal entre las organizaciones es una tendencia creciente, que exige la concepción y el desarrollo de nuevas clases de estrategias (Minztberg y Lampel, 1999). Históricamente el desarrollo de la estrategia de empresa ha estado en función de la competencia entre las organizaciones; cada empresa diseña su estrategia con el propósito de competir con otras, ser rentable y productiva; lo anterior se evidencia en el mantenimiento de ideas como la supervivencia del más fuerte al momento de diseñar las estrategias corporativas (Hakanson y Snehota, 2006).

En el nuevo entorno de negocios caracterizado por el trabajo en red, el desenvolvimiento de cada compañía no puede ser concebido como una actividad individual de cada empresa (Ford y Mouzas, 2008), por esta razón el desarrollo de la estrategia no puede basarse en los esfuerzos que cada una de ellas realice de manera individual para lograr una ventaja competitiva. Tal como lo han señalado Möller y Halinen (1999, citados por Eng, 2005) la planeación estratégica no se constituye en un proceso útil cuando las acciones que emprenden las organizaciones son independientes de las acciones de los otros agentes que conforman la red.

Desde este enfoque, las empresas que hacen parte de una red necesitan también gestionar las interdependencias de actividades y recursos entre los diferentes agentes (Eng, 2005). Tales interdependencias propician la evolución de la estrategia de cada una de las empresas que conforman la red lo que a su vez tiene efectos que facilitan o restringen la evolución de la red como un todo (Ford y Mouzas, 2008).

Adentrarse en la era de la información ha significado para las empresas una serie de ajustes, cambios y retos que les permitan crecer y proyectarse en el futuro, especialmente bajo el nuevo paradigma de trabajo colaborativo entre organizaciones, pese a lo anterior, se ha identificado y discutido de manera limitada en

ISSN: 07I 8-2724. (http://www.jotmi.org)

Journal of Technology Management \& Innovation (C) Universidad Alberto Hurtado, Facultad de Economía y Negocios 
torno a la estrategia de negocios en "condiciones de red" (Hakanson y Snehota, 2006).

Si bien no existe un importante cuerpo de investigación que aporte evidencia empírica en torno al proceso de construcción de estrategia desde el enfoque de red, se han planteado algunos esquemas. Por ejemplo, Hakanson y Snehota (2006) señalan que para la implementación de una estrategia óptima en condiciones de red, se hace necesario llevar a cabo cambios considerables. Tales cambios podrían estar asociados a la forma como asignan y estructuran los recursos internos y en la manera como relacionan sus propias actividades y recursos con los otros agentes que hacen parte de la red.

Con base en la limitada conceptualización y evidencias en torno al concepto de estrategia desde la perspectiva de red, el presente artículo busca realizar un aporte a través del análisis de la forma como las organizaciones que constituyen una red tienen en cuenta a los otros agentes en el proceso de formulación y control de la estrategia empresarial.

\section{Método}

\section{Participantes}

Para la investigación se empleó como muestra un grupo de cinco empresas que desde el año 2006 conforman una red formal de empresas en sector cosmético en Bogotá (Colombia).

La antigüedad promedio de las empresas participantes era de 23,2 años, la más antigua de las empresas contaba con 50 años de existencia y la más joven con II años.

En relación con el género de los gerentes que hicieron parte del estudio, tres eran hombres y dos mujeres. En todos los casos, los gerentes de las empresas participantes del estudio tenían calidad de propietarios o socios. Cuatro de los gerentes de las empresas contaban con estudios universitarios, el quinto gerente, contaba con estudios de bachillerato.

\section{Instrumento}

Para la recolección de la información se estructuró un cuestionario el cual se conformaba de dos partes. La primera parte contenía variables de tipo sociodemográfico como antigüedad en el cargo, género, edad y nivel educativo del gerente de la empresa así como también permitía identificar antigüedad, tipo y tamaño de la empresa.

La segunda parte del cuestionario exploraba aspectos relacionados con la estrategia empresarial. Para tales fines, se construyeron dos bloques de preguntas; en el primer bloque se exploraba la medida en que las organizaciones tenían en cuenta su interacción con otros actores, empresas o instituciones para formular sus estrategias. El segundo bloque a su vez analizaba la medida en que la organización tenía en cuenta su interacción con otros actores, empresas o instituciones para realizar controles y ajustes a la estrategia empresarial.

Para la valoración de los ítems se empleó una escala de 0 a 2 donde 0 significaba ningún grado de consideración, I moderado grado de consideración y 2 alto grado de consideración.

\section{Tipo de estudio}

La investigación se circunscribió a un estudio de carácter no experimental dado que los fenómenos fueron observados de manera natural, sin intervenir en su desarrollo.

Así mismo la investigación se constituyó en un estudio de tipo exploratorio dado existían pocos antecedentes empíricos en torno al tema y se disponía de limitada información tanto de carácter conceptual como metodológico.

\section{Procedimiento}

Para la recogida de información se enviaron los cuestionarios vía correo electrónico, los cuales fueron debidamente diligenciados por los gerentes de cada una de las empresas que componían la muestra.

Para el análisis de los datos se empleó una aproximación desde teoría de grafos, abordando aspectos relacionados con la estructura de la red estudiada. En cuanto a las herramientas computacionales se usó el programa para el análisis de redes Ucinet (Borgatti, et al., 2002).

Para la presentación de los resultados y el análisis de la información se emplearon dos medidas de centralidad en teoría de redes: degree (deg) y eigenvector (ev). La medida

ISSN: 07I 8-2724. (http://www.jotmi.org) 
deg indica el número de conexiones de un nodo en la red y puede tomar valores tantos cuantos contactos tenga con otros nodos; el coeficiente ev mide la importancia de un nodo en la red y toma valores que van de cero a uno; a mayor valor, mayor importancia tiene el nodo en la red.

\section{Resultados}

La presentación de resultados se realiza tomando como base los cinco grupos de agentes incorporados en el estudio: a) los grupos de interés, b) las instituciones del estado de nivel nacional, c) las instituciones del estado de nivel departamental, d) las instituciones del estado de nivel distrital y e) otros actores. Por cada uno de ellos se realiza una descripción de la medida en que las empresas del estudio tienen en cuenta estos agentes para formular $y$ realizar controles a la estrategia empresarial.

\section{Grado de consideración de grupos de interés}

Al analizar el grado en el que las organizaciones tienen en cuenta su relación con los grupos de interés para formular sus estrategias, se observa que en general, el papel de los empleados $(e v=0,345)$ y los consumidores $(e v=0,345)$ son los de mayor representatividad seguidos por el papel de los competidores $(\mathrm{ev}=0,302)$ tal como se ilustra en la Tabla I.

Los resultados indican también que al momento de formular las estrategias organizacionales, las empresas que conforman la red dan un peso importante a las relaciones que han establecido con otras empresas con las que tiene alianzas (ev= 0,302); los últimos lugares son ocupados por los socios $(e v=0,277)$ y las otras empresas que conforman la red $(\mathrm{ev}=0,23 \mathrm{I})$ en su orden (Ver Tabla I).

Complementariamente, al analizar la medida en que las organizaciones consideran los grupos de interés para realizar procesos de control a la estrategia, se observa que las empresas dan mayor valor a la información que proviene de los empleados (ev= 0,33I), los competidores (ev= 0,331 ) y de las empresas que conforman la red en mayor medida (ev=0,33l), tal como se ilustra en la Tabla I.

\begin{tabular}{|l|c|c|c|r|}
\cline { 2 - 5 } \multicolumn{1}{c|}{} & \multicolumn{2}{c|}{ DEGRE } & \multicolumn{2}{c|}{ EIGENVECTOR } \\
\hline Grupos de Interés & Formulación & Control & Formulación & Control \\
\hline Empleados & 5 & 5 & 0.345 & 0.331 \\
\hline Socios & 4 & 4 & 0.277 & 0.269 \\
\hline Consumidores & 5 & 4 & 0.345 & 0.269 \\
\hline Competidores & 4 & 5 & 0.302 & 0.331 \\
\hline Empresas que conforman la red & 3 & 5 & 0.231 & 0.331 \\
\hline $\begin{array}{l}\text { Otras empresas con las que tiene } \\
\text { alianzas }\end{array}$ & 4 & 4 & 0.302 & 0.269 \\
\hline
\end{tabular}

Tabla I. Nivel de consideración de los grupos de interés

En el proceso de control de la estrategia, las empresas reconocen cierto papel, aunque menos representativo para el proceso, a la interacción con los socios (ev= 0,269), los consumidores $(\mathrm{ev}=0,269)$ y las otras empresas con las que tienen alianzas $(\mathrm{ev}=0,269)$.

\section{Grado de consideración de instituciones del estado de nivel nacional}

De otra parte, los resultados también indican que el papel de las instituciones del estado a nivel nacional es poco representativo a la hora de formular estrategias empresariales, tal como se observa en la Tabla 2.

Particularmente se identifica que las organizaciones consideran en mayor medida las entidades de fomento en torno a aspectos financieros (ev= 0,416$)$ seguidas de los ministerios ( $\mathrm{ev}=0,342)$ y las entidades de fomento que promueven aspectos de carácter administrativo (ev= $0,321)$. 


\begin{tabular}{|l|c|c|c|c|}
\cline { 2 - 5 } \multicolumn{2}{c|}{} & \multicolumn{2}{c|}{ DEGREE } & \multicolumn{2}{c|}{ EIGENVECTOR } \\
\hline $\begin{array}{l}\text { Instituciones del Estado a nivel } \\
\text { nacional }\end{array}$ & Formulación & Control & Formulación & Control \\
\hline Ministerios & 4 & 3 & 0.342 & 0.294 \\
\hline Universidades dispectos & 2 & 2 & 0.208 & 0.245 \\
\hline $\begin{array}{l}\text { Entidades de Fomento ácnicos } \\
\text { Ténictos }\end{array}$ & 3 & 3 & 0.311 & 0.316 \\
\hline $\begin{array}{l}\text { Entidades de Fomento aspectos } \\
\text { Financieros }\end{array}$ & 4 & 3 & 0.416 & 0.390 \\
\hline $\begin{array}{l}\text { Entidades de Fomento aspectos } \\
\text { Administrativos }\end{array}$ & 3 & 3 & 0.321 & 0.390 \\
\hline \begin{tabular}{l} 
Entidades de Fomento a la Investigación \\
\hline
\end{tabular} & 1 & 2 & 0.095 & 0.192 \\
\hline
\end{tabular}

Tabla 2. Nivel de consideración de las instituciones del estado de nivel nacional

En torno a la medida en que las empresas atribuyen importancia a las instituciones del estado de nivel nacional para formular estrategias empresariales (Tabla 2), los resultados indican que las entidades de fomento de carácter técnico (ev= $0,3 \mathrm{II})$ se posicionan en cuarto lugar; los dos últimos lugares fueron concedidos a las universidades públicas de nivel nacional $(\mathrm{ev}=0.208)$ y las entidades de fomento a la investigación del nivel nacional $(\mathrm{ev}=0.095)$.

En relación con el papel de las instituciones del estado de nivel nacional en el proceso de control estratégico, los resultados obtenidos indican que las instituciones que promueven y fomentan aspectos de carácter financiero y administrativo $(\mathrm{ev}=0,390)$ son más ampliamente consideradas (Ver Tabla 2). Las entidades de fomento en aspectos técnicos $(\mathrm{ev}=0,316)$ del nivel nacional y los ministerios $(e v=0,294)$ ocupan el tercer y cuarto lugar en esta valoración, no obstante juegan también un papel en este contexto.

En contraste, las entidades de carácter académico e investigativo como las universidades $(\mathrm{ev}=0,245)$ y entidades de fomento a la investigación (ev= 0,192) muestran tener unos valores poco representativos cuando se estudia la importancia de estos actores en el marco del análisis de la red (Tabla 2).

\section{Grado de consideración de instituciones del estado de nivel departamental}

Al analizar el papel de las instituciones del estado a nivel departamental con miras a la formulación de estrategias empresariales, llama la atención que prácticamente no son tenidas en cuenta por las organizaciones que conforman la muestra (ver Tabla 3).

\begin{tabular}{|c|c|c|c|c|}
\hline \multirow[b]{2}{*}{$\begin{array}{l}\text { Instituciones del Estado a nivel } \\
\text { Departamental }\end{array}$} & \multicolumn{2}{|c|}{ DEGREE } & \multicolumn{2}{|c|}{ EIGENVECTOR } \\
\hline & Formulación & Control & Formulación & Control \\
\hline Universidades & 3 & 4 & 0.500 & 0.447 \\
\hline Entidades de Fomento aspectos Técnicos & I & 1 & 0.500 & 0.447 \\
\hline $\begin{array}{lll}\text { Entidades de Fomento aspectos } \\
\text { Financieros }\end{array}$ & $\mathrm{I}$ & I & 0.500 & 0.447 \\
\hline $\begin{array}{lrl}\text { Entidades de Fomento aspectos } \\
\text { Administrativos }\end{array}$ & 1 & 1 & 0.500 & 0.447 \\
\hline Entidades de Fomento a la Investigación & 0 & $\mathrm{I}$ & 0.000 & 0.447 \\
\hline
\end{tabular}

Tabla 3. Nivel de consideración de las instituciones del estado de nivel departamental

ISSN: 07I 8-2724. (http://www.jotmi.org)

Journal of Technology Management \& Innovation (C) Universidad Alberto Hurtado, Facultad de Economía y Negocios 
Los resultados indican que solo una de las organizaciones que participó del estudio reconoce haber tenido en cuenta su interacción con entidades de fomento en aspectos técnicos (deg= I), financieros ( $\operatorname{deg}=1$ ) y administrativos $(\operatorname{deg}=1)$ para determinar su estrategia empresarial.

Nuevamente, las entidades de fomento a la investigación de carácter departamental no juegan ningún papel de cara a la formulación de estrategias entre las organizaciones participantes $(\mathrm{deg}=0 ; \mathrm{ev}=0,00)$ tal como se ilustra en la Tabla 3.

En cuanto al nivel de consideración de las instituciones del estado a nivel departamental (Tabla 3), los resultados indican también que las organizaciones prácticamente no las tienen en cuenta para la realización de procesos de control estratégico. Solamente una de las empresas participantes manifestó considerar este tipo de entidades para dicho proceso aunque el peso que le atribuye es moderado. Particularmente son entidades de fomento en aspectos de carácter técnico $(\mathrm{deg}=\mathrm{I})$, financiero $(\mathrm{deg}=\mathrm{I})$ y administrativo ( $\mathrm{deg}=\mathrm{I})$ las consideradas por esta organización.

\section{Grado de consideración de instituciones del estado de nivel distrital}

Al considerar el grado en que las organizaciones tienen en cuenta su interacción con entidades del estado a nivel distrital para formular sus estrategias empresariales, los resultados señalan que las entidades de fomento en aspectos administrativos $(\mathrm{ev}=0,418)$, técnicos $(\mathrm{ev}=$ $0,373)$ y financieros (ev= 0,373) son más ampliamente consideradas en contraste con las entidades de nivel departamental (Tabla 4).

\begin{tabular}{|l|c|r|c|c|}
\cline { 2 - 6 } \multicolumn{2}{c|}{} & \multicolumn{2}{c|}{ DEGREE } & \multicolumn{2}{c|}{ EIGENVECTOR } \\
\hline $\begin{array}{l}\text { Instituciones del estado a nivel } \\
\text { distrital }\end{array}$ & Formulación & Control & Formulación & Control \\
\hline $\begin{array}{l}\text { Entidades de Fomento aspectos } \\
\text { Técnicos }\end{array}$ & 3 & 3 & 0.373 & 0.338 \\
\hline $\begin{array}{l}\text { Entidades de Fomento aspectos } \\
\text { Financieros }\end{array}$ & 3 & 4 & 0.373 & 0.415 \\
\hline $\begin{array}{l}\text { Entidades de Fomento aspectos } \\
\text { Administrativos }\end{array}$ & 4 & 4 & 0.418 & 0.415 \\
\hline \begin{tabular}{l} 
Entidades de Fomento a la Investigación \\
\hline
\end{tabular} & 0 & 1 & 0.000 & 0.120 \\
\hline
\end{tabular}

Tabla 4. Nivel de consideración de las instituciones del estado de nivel distrital

Los resultados obtenidos también indican que las entidades de fomento a la investigación en el ámbito distrital no son tenidas en cuenta al momento de establecer las estrategias empresariales $(\mathrm{deg}=0 ; \mathrm{ev}=0,00)$ tal como se ilustra en la Tabla 4.

Con base en los resultados, es posible indicar además que las instituciones del estado de nivel distrital parecen tener mayor representatividad entre las empresas al momento de realizar procesos de control estratégico en comparación con las entidades de nivel departamental. En este caso, las empresas que conformaban la muestra del estudio indicaron que las entidades de fomento en aspectos financieros $y$ en aspectos administrativos (ev=
$0,415)$ son consideradas en alguna medida para efectos de realizar controles a la estrategia empresarial (Ver Tabla 4).

Así mismo los resultados señalan que las entidades de nivel distrital que promueven aspectos de carácter técnico en las organizaciones $(\mathrm{ev}=0,338)$ son consideradas por las empresas en el proceso de control estratégico; en contraste, las instituciones de fomento a la investigación de carácter distrital $(e v=0,120)$ son consideradas en escasa o casi nula medida, tal como se ilustra en la Tabla 4.

\section{Grado de consideración de otros actores}

Al analizar el nivel de consideración de otros actores de cara a la formulación de estrategias empresariales, se 
observa que para la mayoría de las organizaciones, la sociedad juega un papel muy importante $(\mathrm{ev}=0,509)$, en contraposición a los organismos multilaterales $(\mathrm{ev}=0,154)$, los cuales prácticamente no son tenidos en cuenta en este proceso, según se ilustra en la Tabla 5.

\begin{tabular}{|l|c|c|c|r|}
\cline { 3 - 6 } \multicolumn{2}{c|}{} & \multicolumn{2}{c|}{ DEGREE } & \multicolumn{2}{c|}{ EIGENVECTOR } \\
\hline Otros actores & Formulación & Control & Formulación & Control \\
\hline $\begin{array}{l}\text { Entidades de Fomento Organismos } \\
\text { Multilaterales }\end{array}$ & & & & \\
\hline La sociedad & 1 & 0 & 0.154 & 0.000 \\
\hline
\end{tabular}

Tabla 5. Nivel de consideración de otros actores

Finalmente, al analizar el nivel de consideración de otros actores en torno al proceso de control estratégico, se observa que los organismos multilaterales no son tenidos en cuenta en este proceso $(\mathrm{ev}=0,000)$ a diferencia de la sociedad (ev= 0,447$)$, que en casi todos los casos se constituye en un elemento de consideración a nivel alto y moderado, tal como se ilustra en la Tabla 5.

En resumen, el mayor peso de cara a los procesos de formulación y control de la estrategia empresarial es otorgado a la interacción con los grupos de interés, seguida de las entidades del estado de nivel distrital. Las entidades del estado de nivel nacional y departamental, tienen un papel poco representativo para las empresas a la hora de realizar controles a sus estrategias empresariales.

\section{Discusión}

Teóricamente se ha señalado que desde la perspectiva de trabajo en red, la formulación y control de la estrategia empresarial se constituye en un proceso dinámico, fundamentado en acciones colectivas, debido a las relaciones de interdependencia y a los procesos de coevolución que se da entre las empresas que conforman una red (Eng, 2005). No obstante, los resultados obtenidos en el presente estudio señalan que no necesariamente existen relaciones de interdependencia entre los agentes de una red $y$ que si estas se presentan, algunas de ellas se establecen en grados variados $y$ en sentidos no siempre justificables.

Los resultados obtenidos en la presente investigación llevan a concluir que las organizaciones, aún perteneciendo a una red empresarial que ha sido constituida hace más de dos años, continúan formulando y controlando sus estrategias empresariales desde un enfoque tradicional, es decir, considerando fundamentalmente su relación con los consumidores y los empleados de la empresa y en menor proporción, teniendo en cuenta las interdependencias con los socios, las otras empresas que conforman la red, las empresas con las que tienen alianzas y los competidores.

En torno a la planeación estratégica desde la perspectiva de red, Möller y Halinen (1999) señalan que este no es un proceso útil en los casos en que las organizaciones emprenden acciones de manera independiente de los otros agentes que conforman la red. En consecuencia, si bien se esperaría que las organizaciones que hacen parte de una red tengan en consideración muy especialmente su interacción con las otras empresas participantes de este proceso asociativo, la evidencia empírica señala que tal condición no se cumple en el marco del presente estudio.

En los resultados obtenidos llama la atención que las empresas que conforman la red dan un valor importante a la interacción que tienen con organizaciones aliadas para determinar sus estrategias empresariales, muy por encima del valor que atribuyen a su interacción con las otras empresas que conforman la red. Pese a que se ha indicado que el trabajo de tipo cooperativo implicaría definición de estrategias cooperativas con todos los agentes implicados, la evidencia empírica indica que no en todos los casos se cumple $\mathrm{o}$ al menos no en la misma proporción.

Una de las explicaciones que se podría dar al fenómeno observado en esta investigación es que si bien la red empresarial que hace parte del estudio se conformó hace más de dos años, es posible que la construcción de lazos de confianza entre sus agentes (en este caso con las otras 
empresas que conforman la red) se encuentre aún en proceso; en contraposición, es probable que los lazos establecidos con otras organizaciones aliadas sean tan fuertes y de tanta trayectoria, que por esta razón se constituyen en agentes representativos para las empresas al momento de formular sus estrategias. Si bien se ha mencionado que el establecimiento de contactos colaborativos entre organizaciones exige el desarrollo de nuevas clases de estrategia (Mintzberg y Lampel, 1999), los resultados obtenidos indican que el camino está aún por recorrerse.

La evidencia obtenida en la investigación señala también que las organizaciones participantes en general, consideran aunque en menor proporción, su interacción con las entidades de fomento en asuntos técnicos, financieros y administrativos tanto de nivel nacional como departamental y distrital. Desde el enfoque de red se ha puesto de manifiesto que es prioritario el papel de las instituciones locales y de apoyo como los gobiernos, las instituciones financieras, los centros de desarrollo empresarial y de servicios tecnológicos debido a que tienen la responsabilidad de regular, financiar, formar los recursos humanos y contribuir al desarrollo tecnológico de las empresas y de las redes (López, 2003); en el presente estudio pese a que dicha relación existe, no es lo suficientemente representativa.

En complemento de lo anterior llama la atención también que las universidades y las instituciones de fomento a la investigación de nivel nacional, departamental o distrital, tienen un papel poco significativo en los procesos de formulación y control de las estrategias empresariales; lo anterior se encuentra en consonancia con la idea que existe una brecha entre la academia y la empresa, lo que en definitiva tiene impacto en la introducción de innovaciones y en los indicadores de productividad $y$ competitividad.

Desde la perspectiva de los sistemas de innovación, Cimoli y De la Mothe (200I) destacan la importancia de pertenecer a una red dado que estas potencian o limitan las capacidades de las empresas para solucionar problemas. Desde esta óptica, el hecho que las empresas del estudio consideren en limitada medida a otros actores $y$ a las instituciones del estado a nivel nacional, departamental y distrital de cara a los procesos de formulación y control de sus estrategias empresariales, podría tener un impacto negativo en los procesos de innovación; sería necesario validar este supuesto en el marco de otra investigación.

El establecimiento de relaciones con agentes externos a las empresas es decir, con otras empresas que conforman la red, empresas aliadas, proveedores, consumidores e instituciones del estado de distintos niveles incluidos centros de investigación y universidades, además de potenciar el aprendizaje interactivo promueven la introducción de innovaciones (Lucendo, 2007). Los resultados de la investigación señalan sin embargo que si bien estas interacciones entre los agentes se dan, no son concebidas desde la perspectiva estratégica. Sería pertinente analizar si al modificar el nivel de consideración de otros agentes al momento de formular $y$ controlar las estrategias empresariales, tendría impacto en la introducción de innovaciones.

Una de las condiciones para que las relaciones establecidas entre los agentes de una red tengan potencial generativo es la direccionalidad alineada, entendida esta como la alineación estratégica hacia un mismo propósito (Lane y Maxfield, 1997). En el estudio realizado es claro que las empresas que conforman la red han establecido un interés común al participar en el emprendimiento de una nueva empresa o spin off, no obstante al analizar la medida en que las empresas tienen en cuenta a los otros agentes para formular y controlar sus estrategias dicho sentido de direccionalidad alineada no es tan claro.

Dado que en el estudio se puso de manifiesto que las empresas consideran de manera limitada a otros agentes de cara a procesos estratégicos, es preciso indagar las razones. Por ejemplo, sería válido determinar si este fenómeno se da por desconocimiento de las empresas, debido a dificultades para acceder a este tipo de instituciones, debido a que las instituciones de apoyo no cuentan con los suficientes mecanismos para promover el fortalecimiento de las redes o porque no disponen de los medios necesarios para dar a conocer que están preparados para apoyar decididamente el crecimiento y desarrollo empresarial.

Como bien se ha evidenciado, la noción de construcción de estrategia desde la perspectiva de redes se encuentra aún en estado germinal, por lo que el presente estudio se constituye en una primera aproximación en el campo. No obstante, es preciso mencionar que la investigación también cuenta con algunas limitaciones. En primer lugar,

ISSN: 07I 8-2724. (http://www.jotmi.org)

Journal of Technology Management \& Innovation (c) Universidad Alberto Hurtado, Facultad de Economía y Negocios 
por tratarse de un estudio exploratorio con una muestra poblacional reducida, los resultados deben ser asumidos con cautela; sería necesario analizar el comportamiento en un número mayor de redes empresariales con el fin de disponer de más elementos a partir de los cuales llegar a conclusiones.

De otra parte se podría llevar a cabo un estudio en el que se analice el impacto que tiene para las organizaciones considerar a los otros agentes tanto en la formulación como en el control de la estrategia empresarial. En dicho análisis se podrían considerar indicadores como innovación, productividad, competitividad, entre otros.

Así mismo, se podría analizar si existen diferencias en los procesos de formulación y control de la estrategia empresarial en función de sectores económicos o sectores estratégicos o tamaños de red.

\section{Referencias}

BARABÁSI, A. L (2003). Linked: Th, New Science of Networks. Penguin Group, New York.

BORGATTI, S.P., Everett, M.G., Freeman, L.C. (2002). Ucinet for Windows: Software for Social Network Analysis. Harvard, MA: Analytic Technologies.

BOGARTI, S. P. (2002). Netdraw Network Visualization. Analytic Technologies, Harvard M.A.

CEGLIE, G., Dini, M. (1999). SME Cluster and Network development in Developing Countries: The experience of UNIDO. PSD TECHNICAL WORKING PAPERS SERIES: Working Paper No.2.

CIMOLI, M., De La Mothe, J. (200I). Technology, Growth and Development: The dynamics of catching up, falling venid and leaping ahead, En J. de la Mothe (ed): Sience, Technology and Governance, Continuum-Pinter Publishers: London, Pp. I53-I72.

COOK, K. S., Emerson, R. M. (1978). Power, Equity and commitment in exchange networks. American Sociological Review, 43, 721-739.

DÍAZ, J. J. (2005). Programa de Desarrollo Empresarial Sectorial PRODES EN Medellín y su área metropolitana en
Asociatividad Empresarial en Colombia. R. Valencia \& J. Muñoz (Compiladores), 16I-168.

DINI, M., Ferraro, C., Gasaly, C. (2007). Pymes y articulación productiva. Resultados y lecciones a partir de experiencias en América Latina. Unidad de Desarrollo Industrial y Tecnológico, Serie Desarrollo Productivo 180, Santiago, Publicación de las Naciones Unidas.

ENG, T. (2005) Conceptualization of strategy in business networks: An assessment. Journal of Marketing Channels, I 3 (I), 79-92.

FORD, D., Mouzas, S. (2008). Is there any hope? The idea of strategy in business networks. Australasian Marketing Journal, 16 (I), 64-78.

FRÍAS, J. C., Rodríguez, N. (2005). Mejoramiento de la productividad de fábricas productoras de muebles de madera. Área Metropolitana. En Asociatividad Empresarial en Colombia. R. Valencia \& J. Muñoz (Compiladores), I5I-I59.

HAKANSSON, H., Snehota, I. (2006). No business is an island: $\mathrm{Ne}$ network concept of business strategy. Scandinavian Journal of Management, 22, 256-270.

LANE, D.A., Maxfield, R. (1997). Foresight Complexity and Strategy, en W.B. Arthur, S. Durlauf and D.A. Lane (eds.), The economy as an evolving complex system II. Redwood City: Addison Wesley, pp. 169-198.

LIPNACK, J., Stamps, J. (1994). The Age of the Network: Organizing Principles for the 2 lst Century. Essex Junction, Vermont: Omino.

LÓPEZ Cerdán, C (2003). Redes empresariales: experiencias en la Región Andina. Manual para el articulador. Minka, Perú.

LUCENDO Monedero, A. L. (2007). Cadenas Productivas e Innovación en el marco territorial andaluz, Consejo Económico y Social de Andalucía: Sevilla.

MINTZBERG, H., Lampel, J. (1999). Reflecting on strategy process, Sloan Management Review, 40 (3), 21-30.

MÖLLER, K. K., Halinen, A. (1999). Business relationships and networks: Managerial challenge of network era. Industrial Marketing Management, 28, 4I3-427. 